\title{
Fully Probabilistic Design for Stochastic Discrete System with Multiplicative Noise
}

\author{
Yuyang Zhou ${ }^{1}$, Randa Herzallah ${ }^{2}$ and Ana Zafar ${ }^{3}$
}

\begin{abstract}
In this paper, a novel algorithm based on fully probabilistic design (FPD) is proposed for a class of linear stochastic dynamic processes with multiplicative noise. Compared with the traditional FPD, the new procedure is presented to deal with multiplicative noise and the system parameters are estimated online by the linear optimisation. The performance index is characterised by the Kullback-Leibler divergence (KLD). The generalised probabilistic control law is obtained by solving the Riccatti equation while taking the multiplicative noise into consideration. To demonstrate the effectiveness of the proposed method, a numerical example is given in comparison with the traditional FPD.
\end{abstract}

\section{INTRODUCTION}

In recent decades, stochastic control has been one of the major interesting research subjects due to the inevitable noises, including external disturbance and structure randomness uncertainties have widely existed in the industrial and chemical system [1]. So far, amounts of research literature related to the stochastic systems control have been published, such as linear quadratic Gaussian (LQG) algorithm [2], minimum variance control [3], [4], minimum entropy control [5]-[7], and $H_{2} / H_{\infty}$ control [8], [9]. Most of the existing literature focus on minimising the system randomness or make the Probability density function (pdf) of the concerned value follow the desired pdf. The quadratic distance has been used in many papers to characterise the distance between the actual pdf and the desire pdf [10]-[12]. As many distributions can be expressed by an exponential function, KullbackLeibler divergence (KLD) can be considered as a convenient scale to characterise the distance between two distributions [13]-[15]. Therefore, Fully Probabilistic Design (FPD) ( [13]-[16]), inspired by Bayesian approach to adaptive control design [17], which is proposed based on KLD, is applied in this paper.

The main contribution of the FPD is that it offers a specific form of the randomised optimal controller. However, the computational loads are quite heavy since the evaluation of the randomised optimal controller involves multivariate integration steps which need to be calculated by backward

\footnotetext{
*The work was funded by a Leverhulme Trust Research Project Grant number RPG-2017-337

${ }^{1}$ Yuyang Zhou is with the Systems Analytics Research Institute, Aston University, Aston Triangle, Birmingham B4 7ET, UK annamada@163.com

2 Randa Herzallah is with the Systems Analytics Research Institute, Aston University, Aston Triangle, Birmingham B4 7ET, UK r.herzallaheaston.ac.uk

3 Ana Zafar is with the Systems Analytics Research Institute, Aston University, Aston Triangle, Birmingham B4 7ET, UK zafara7@aston.ac.uk
}

recursion. To solve that, [14], [18] provides a probabilistic Dual Heuristic Programming (DHP) adaptive critic method, which applies a critic network to circumvent the requirement for explicitly evaluating the optimal value function and enormously reduced computational requirements.

Several numbers of studies based on FPD has been published and gain significant accomplishments. A novel generalised fully probabilistic controller design was proposed in [15] for stochastic linear Gaussian systems where the dynamics of the system is unknown and where the uncertainty introduced by the model discrepancy is estimated as a function of the system inputs. In [19], the FPD is combine with distributed control for large, complex, noisy and highly connected systems. In [20], a probabilistic control method for adaptive synchronisation is proposed. So far, very little published FPD related literature has focused on the system with multiplicative noise, which is commonly existed in many physical systems such as biological movement systems [20], [21] and signal processing systems [22], [23]. Compared with additive noise, multiplicative noise is considered to be more practical as it enables the statistical description of the noise part to be uncertain but at the same time depends on the control and state solution [24]. Moreover, multiplicative noise in a linear control problem has a crucial effect on the robustness of the control system [24], [25]. Therefore, it is significant to develop an FPD method to deal with stochastic systems with multiplicative noise.

This paper proposes an FPD based controller for a class of stochastic linear discrete system with multiplicative noise to make sure the states pdf approaching the given pdf. Compared with the traditional FPD, multiplicative noise is considered in this paper and the state parameters are estimated by a single layer neural network. In addition, the state feedback controller form is applied. As a traditional control methodology, state feedback control has always played an important role in both theoretical and practical fields. The way of choosing the optimal feedback gain is various with the performance index, such as LQR [26]-[28] and pole region assignment [29]. Also, state feedback control draws loads of attention in stochastic system control. The problem of designing $H_{\infty}$ state feedback controller for the generalized time-delay systems with delayed states and control inputs in continuous and discrete time cases is considered in [30]. Two novel methods for stochastic systems using the state feedback form were proposed in [3] and [6], where [3] is with minimum entropy based control and [6] minimum variance based control. However, the optimal feedback gain in most existing stochastic related literature has no explicit form, 
which makes it hard to achieve a global optimal solution. Motivated by that, in this paper, the explicit form of feedback gain is provided and the detailed procedure is given.

The remainder of this paper is organised as follows. Section I indicates the problem statement. Section II provides the details of the controller design. In Section III, the proposed algorithm is applied to a numerical example to show its effectiveness. Finally, the conclusion is summarised in Section IV.

\section{Problem Statement}

\section{A. Model Description}

Consider a linear discrete time system with multiplicative Gaussian noise as follows

$$
x_{k}=\bar{A} x_{k-1}+\bar{B} u_{k-1}+\bar{D} x_{k-1} v_{k-1}
$$

where $x_{k} \in \mathfrak{R}^{n}$ is the system states, $u_{k} \in \mathfrak{R}^{m}$ is the system inputs, $\bar{A}, \bar{B}, \bar{D}$ are system matrices with appropriate states dimensions, and $v_{k} \in \mathfrak{R}$ is independent Gaussian noise with zero mean and variance $\bar{Q}$

$$
v_{k} \sim N(0, \bar{Q})
$$

This means that the conditional distribution of the system dynamics described in Eq.(1) is a Gaussian distribution with mean $\mu_{k}$ and covariance $\Sigma_{k}$,

$$
s\left(x_{k} \mid u_{k-1}, x_{k-1}\right) \sim N\left(\bar{\mu}_{k}, \bar{\Sigma}_{k}\right)
$$

where

$$
\begin{aligned}
\bar{\mu}_{k} & =\bar{A} x_{k-1}+\bar{B} u_{k-1} \\
\bar{\Sigma}_{k} & =\operatorname{cov}\left(x_{k} \mid u_{k-1}, x_{k-1}\right) \\
& =E\left\{\left(x_{k}-\mu_{k}\right)\left(x_{k}-\mu_{k}\right)^{T}\right\} \\
& =E\left\{\bar{D} x_{k-1} v_{k-1} v_{k-1}^{T} x_{k-1}^{T} \bar{D}^{T}\right\} \\
& =\bar{D} x_{k-1} \bar{Q} x_{k-1}^{T} \bar{D}^{T}
\end{aligned}
$$

\section{B. Parameter Estimation}

A key step in the fully probabilistic design is the evaluation of the probabilistic model of the discrete time linear invariant stochastic dynamical system. The system considered in this paper is described by the state space model shown in Eq.(1). The previous state and control input do not entirely determine the present state due to the multiplicative noise. However, the state and control input of the previous state allow the specification of the probability distribution of the states, given by $s\left(x_{k} \mid u_{k-1}, x_{k-1}\right)$. To predict the unknown probabilistic distribution, the parameters of the distribution of system (1) are estimated by the implementation of linear optimisation. The linear optimisation problem is conducted in two parts:

- For the probabilistic model, the parameters, $\bar{A}$ and $\bar{B}$, in Eq.(1) are unknown and need to be estimated as $A$ and $B$. The estimations are obtained by rearranging Eq.(1) and linearly optimizing as

$$
x_{k}-\bar{D} x_{k-1} v_{k-1}=A x_{k-1}+B u_{k-1} .
$$

Note that Eq.(6) is written in matrix format and the right hand side of Eq.(6) is written as:

$$
\left[\begin{array}{cccc}
a_{11} & \ldots & a_{1 n} & b_{11} \\
\vdots & \ddots & \vdots & \vdots \\
a_{n 1} & \ldots & a_{n n} & b_{n 1}
\end{array}\right]\left[\begin{array}{c}
x_{k-1} \\
u_{k-1}
\end{array}\right]
$$

where the first matrix is the combined matrix of $A$ and $B$ of the probabilistic model which can be split accordingly in two separate matrices $A$ and $B$. To solve this linear optimisation problem and obtain our estimations for $A$ and $B$, the pseudo inverse of $\left[\begin{array}{l}x_{k-1} \\ u_{k-1}\end{array}\right]$ will be taken, resulting in,

$$
\left[x_{k}-\bar{D} x_{k-1} v_{k-1}\right] * \text { pseudoinverse }\left[\begin{array}{l}
x_{k-1} \\
u_{k-1}
\end{array}\right] \text {. }
$$

- Similarly, matrix $D$ is estimated by rearranging (1), and thus, linearly optimizing $x_{k}-\bar{A} x_{k-1}-\bar{B} u_{k-1}=D x_{k-1} v_{k-1}$. The pseudo inverse in this case is taken of $x_{k-1} v_{k-1}$.

This yield the following estimated distribution of the stochastic system described by Eq.(1),

$$
s\left(x_{k} \mid u_{k-1}, x_{k-1}\right) \sim N\left(\mu_{k}, \Sigma_{k}\right)
$$

where,

$$
\begin{aligned}
& \mu_{k}=A x_{k-1}+B u_{k-1} \\
& \Sigma_{k}=D x_{k-1} \bar{Q} x_{k-1}^{T} D^{T}
\end{aligned}
$$

\section{Performance Index}

The purpose of designed controller here is to design a control strategy for the system in Eq.(1) to bring all the initial states back to the origin, in the meantime making sure the state conditional pdf follows a predefined pdf. Therefore, the performance index needs to be formed to indicate the distance between the state conditional pdf and the desired pdf. There are various methods to describe the distance between two distributions, such as Kullback-Leibler divergence (KLD) and Cauchy-Schwarz (CS). In this paper, the KLD is applied due to its computational advantage. The definition of the KullbackLeibler divergence (KLD) between the actual joint pdf $f(D)$ of the observed data $D=(x(H), u(H))$ and the ideal joint pdf $f^{I}(D)$ on a set of possible $D$ s is given by

$$
D\left(f \| f^{I}\right)=\int f(D) \ln \left(\frac{f(D)}{f^{I}(D)}\right) d D
$$

Based on the chain rule for pdfs [31], with $\mathrm{H}$ being the control horizon, the probabilistic closed-loop description of the system dynamics could be evaluated as follows:

$$
f(D)=\prod_{k=1}^{H} s\left(x_{k} \mid u_{k-1}, x_{k-1}\right) c\left(u_{k-1} \mid x_{k-1}\right) .
$$

Similarly, the ideal probabilistic closed-loop pdf can be expressed in the same form as Eq.(11) with ideal system model pdf $s^{I}\left(x_{k} \mid u_{k-1}, x_{k-1}\right)$ and ideal controller pdf $c^{I}\left(u_{k-1} \mid x_{k-1}\right)$,

$$
f^{I}(D)=\prod_{k=1}^{H} s^{I}\left(x_{k} \mid u_{k-1}, x_{k-1}\right) c^{I}\left(u_{k-1} \mid x_{k-1}\right) .
$$


With the KL-distance (10), the closed loop joint pdf (11) and the desire closed loop joint pdf (12), the performance index can be formalised to be given by the following expression:

$$
\begin{aligned}
-\ln \left(\gamma\left(x_{k-1}\right)\right)= & \min _{\left\{c\left(u_{k-1} \mid x_{k-1}\right)_{\tau \geq k}\right\}} \sum_{\tau=k}^{H} \int f\left(d_{\tau} \mid x_{k-1}\right) \\
& \times \ln \left(\frac{s\left(x_{\tau} \mid u_{\tau-1}, x_{\tau-1}\right) c\left(u_{\tau-1} \mid x_{\tau-1}\right)}{s^{I}\left(x_{\tau} \mid u_{\tau-1}, x_{\tau-1}\right) c^{I}\left(u_{\tau-1} \mid x_{\tau-1}\right)}\right) \\
& \times d\left(d_{\tau}\right),
\end{aligned}
$$

For arbitrary $\tau \in\{1, \ldots, H\}$. Using the dynamic programming (DP) concept [32], Eq.(13) can be rewritten in the following recurrence functional form:

$$
\begin{aligned}
-\ln \left(\gamma\left(x_{k-1}\right)\right)= & \min _{c\left(u_{k-1} \mid x_{k-1}\right)} \int s\left(x_{k} \mid u_{k-1}, x_{k-1}\right) c\left(u_{k-1} \mid x_{k-1}\right) \\
& \times\left[\ln \left(\frac{s\left(x_{k} \mid u_{k-1}, x_{k-1}\right) c\left(u_{k-1} \mid x_{k-1}\right)}{s^{I}\left(x_{k} \mid u_{k-1}, x_{k-1}\right) c^{I}\left(u_{k-1} \mid x_{k-1}\right)}\right)\right. \\
& \left.-\ln \left(\gamma\left(x_{k}\right)\right)\right] d\left(x_{k}, u_{k-1}\right),
\end{aligned}
$$

where the first item in parenthesis in Eq.(14) represents the partial cost while the second item is the expected minimum cost-to-go function.

The recursive formulation of performance index (14) is similar to Dynamic programming. Full derivation of Eq.(14) can be found in [14]

\section{Optimal Controller Law}

Based on the Fully Probabilistic Design (FPD) [13][15], the control law $c^{*}\left(u_{k-1} \mid x_{k-1}\right)$ which minimises the performance index (14) takes the following form,

$$
\begin{aligned}
& c^{*}\left(u_{k-1} \mid x_{k-1}\right)= \\
& \frac{c^{I}\left(u_{k-1} \mid x_{k-1}\right) \exp \left[-\beta_{1}\left(u_{k-1}, x_{k-1}\right)-\beta_{2}\left(u_{k-1}, x_{k-1}\right)\right]}{\gamma\left(x_{k-1}\right)}
\end{aligned}
$$

where

$$
\begin{aligned}
\gamma\left(x_{k-1}\right)= & \int c^{I}\left(u_{k-1} \mid x_{k-1}\right) \exp \left[-\beta_{1}\left(u_{k-1}, x_{k-1}\right)\right. \\
& \left.-\beta_{2}\left(u_{k-1}, x_{k-1}\right)\right] d u_{k-1} \\
\beta_{1}\left(u_{k-1}, x_{k-1}\right)= & \int s\left(x_{k} \mid u_{k-1}, x_{k-1}\right)\left[\ln \frac{s\left(x_{k} \mid u_{k-1}, x_{k-1}\right)}{s^{I}\left(x_{k} \mid u_{k-1}, x_{k-1}\right)}\right] d x_{k} \\
\beta_{2}\left(u_{k-1}, x_{k-1}\right)= & -\int s\left(x_{k} \mid u_{k-1}, x_{k-1}\right) \ln \left(\gamma\left(x_{k}\right)\right) d x_{k}
\end{aligned}
$$

Remark 1: The optimal controller law (15) for minimisation of KLD (13) is not just for linear discrete system (1) but for more general nonlinear systems governed by the following nonlinear equation $x_{k}=g\left(x_{k-1}, u_{k-1}, w_{k}\right)$ whose state distribution can be described by $s\left(x_{k} \mid u_{k-1}, x_{k-1}\right)$ and the conditional controller distribution can be described by $c\left(u_{k-1} \mid x_{k-1}\right)$. Note that the optimal form that Eq.(16) offers is basicly a distribution. The actual parameters of the controller distribution can then be obtained based on (16) and the details will be given in the next section.

\section{Algorithm Design}

In this section the generalised fully probabilistic control solution of the regulation problem for the stochastic linear system with multiplicative noise is derived. The purpose of the designed controller here is to make the state pdf $s\left(x_{k} \mid u_{k-1}, x_{k-1}\right)$ follows the given pdf $s^{I}\left(x_{k} \mid u_{k-1}, x_{k-1}\right)$ and return the system states to zero, which is called regulation of dynamical systems. Since a regulation problem, the ideal distribution of the system described by Eq.(1) is specified as

$$
s^{I}\left(x_{k} \mid u_{k-1,} x_{k-1}\right) \sim N\left(0, \Sigma_{2}\right)
$$

where $\Sigma_{k}-\Sigma_{2}$ is assumed to be a positive definite matrix, meaning that the variance of system is decreased and the system has less randomness.

The conditional pdf of the controller can be also charactered as $c\left(u_{k-1} \mid x_{k-1}\right)$, whose ideal distribution is taken to be Gaussian with the following form

$$
c^{I}\left(u_{k-1} \mid x_{k-1}\right) \sim N(0, \Gamma)
$$

where $\Gamma$ is the ideal covariance of input, which indicates the allowed range of optimal control input.

As will be seen from further developments, the solution of Eq.(15) and Eq.(16) subject to the previously described system pdfs Eq.(7), Eq.(17), and Eq.(18) yields the optimal performance index specified by the following theorem.

Theorem 1: Using the pdf description of the system dynamics specified by Eq.(3), the ideal distribution of the system dynamics given by Eq.(17) and the ideal distribution of the controller given by Eq.(18) in Eq.(15) and Eq.(16) yield the following performance index,

$$
-\ln \left(\gamma\left(x_{k}\right)\right)=0.5 x_{k}^{T} S_{k} x_{k}+0.5 w_{k}
$$

where,

$$
\begin{aligned}
S_{k-1} & =-A^{T}\left(\Sigma_{2}^{-1}+S_{k}\right) B\left(B^{T}\left(\Sigma_{2}^{-1}+S_{k}\right) B+\Gamma^{-1}\right)^{-1} B^{T} \\
& \times\left(\Sigma_{2}^{-1}+S_{k}\right)^{T} A+A^{T}\left(\Sigma_{2}^{-1}+S_{k}\right) A+M_{2} \\
w_{k-1} & =\omega_{k}+\ln (2 \Gamma) \\
& +\ln \left(0.5\left(B^{T}\left(\Sigma_{2}^{-1}+S_{k}\right) B+\Gamma^{-1}\right)\right)
\end{aligned}
$$

and where,

$$
M_{2}=D^{T} S_{k} \bar{Q} D
$$

Proof: The quadratic form of the optimal performance function specified in Eq.(19) can be verified by evaluating $\gamma$ in Eq.(17), repeated here,

$$
\gamma\left(x_{k-1}\right)=\int c^{I} \exp \left(-\beta_{1}-\beta_{2}\right) d u_{k-1}
$$

This evaluation requires the evaluation of $\beta_{1}$ and $\beta_{2}$. Starting 
with $\beta_{1}$

$$
\begin{aligned}
& \beta_{1}\left(u_{k-1}, x_{k-1}\right)=\int s\left(x_{k} \mid u_{k-1}, x_{k-1}\right) \ln \frac{s\left(x_{k} \mid u_{k-1}, x_{k-1}\right)}{s^{I}\left(x_{k} \mid u_{k-1}, x_{k-1}\right)} d x_{k} \\
& =\int N\left(\mu_{k}, \Sigma_{k}\right) \ln \frac{N\left(\mu_{k}, \Sigma_{k}\right)}{N\left(0, \Sigma_{2}\right)} d x_{k} \\
& =\int N\left(\mu_{k}, \Sigma_{k}\right)\left(-0.5 \ln \left(\left|\Sigma_{k}\right|\left|\Sigma_{2}\right|^{-1}\right)\right. \\
& -\left(x_{k}-\left[A x_{k-1}+B u_{k-1}\right]\right)^{T}\left(2 \Sigma_{k}\right)^{-1} \\
& \left.\times\left(x_{k}-\left[A x_{k-1}+B u_{k-1}\right]\right)-x_{k}^{T}\left(2 \Sigma_{2}\right)^{-1} x_{k}\right) d x_{k} .
\end{aligned}
$$

To solve Eq.(24), the following rule from [30] is required with positive defined matrix $A_{1}$

$$
\ln \left(\operatorname{det}\left(A_{1}\right)\right)=\operatorname{tr}\left(\log \left(A_{1}\right)\right)
$$

Therefore, the $\ln \left(\left|\Sigma_{k}\right|\left|\Sigma_{2}\right|^{-1}\right)$ term in Eq.(24) can be rewritten as

$$
\begin{aligned}
\ln \left(\left|\Sigma_{k}\right|\left|\Sigma_{2}\right|^{-1}\right) & =\ln \left(\left|\Sigma_{k} \Sigma_{2}^{-1}\right|\right) \\
& =\operatorname{tr}\left(\log \left(\Sigma_{k} \Sigma_{2}^{-1}\right)\right)
\end{aligned}
$$

Assumption 1: For the considered regulation problem, it is expected that at steady state the covariance of the system dynamics will become close to the covariance of the specified ideal distribution. This means that,

$$
\left\|\Sigma_{k} \Sigma_{2}^{-1}-I\right\|<1
$$

then based on lemma 2.6 in [33], Eq.(26) can be approximated as follows,

$$
\operatorname{tr}\left(\log \left(\Sigma_{k} \Sigma_{2}^{-1}\right)\right) \approx \operatorname{tr}\left(\Sigma_{k} \Sigma_{2}^{-1}-I\right) \approx \operatorname{tr}\left(\Sigma_{k} \Sigma_{2}^{-1}\right)-n
$$

where $\mathrm{n}$ is the dimension of $\mathrm{x}$.

Using Eq.(28) in Eq.(24) and expanding the terms of Eq.(24) we get,

$$
\begin{aligned}
& \beta_{1}\left(u_{k-1}, x_{k-1}\right)=\int N\left(\mu_{k}, \Sigma_{k}\right)\left(-0.5 \operatorname{tr}\left(\Sigma_{k} \Sigma_{2}^{-1}\right)+0.5 n\right. \\
& +0.5 x_{k}^{T}\left(\Sigma_{2}^{-1}-\Sigma_{k}^{-1}\right) x_{k}-0.5\left[A x_{k-1}+B u_{k-1}\right]^{T} \Sigma_{k}^{-1} \\
& \left.\times\left[A x_{k-1}+B u_{k-1}\right]+x_{k}^{T} \Sigma_{k}^{-1}\left[A x_{k-1}+B u_{k-1}\right]\right) d x_{k} \\
& =0.5\left[A x_{k-1}+B u_{k-1}\right]^{T} \Sigma_{k}^{-1}\left[A x_{k-1}+B u_{k-1}\right] \\
& -0.5 \operatorname{tr}\left(\Sigma_{k} \Sigma_{2}^{-1}\right)+0.5 n+0.5 \int N\left(\mu_{k}, \Sigma_{k}\right) x_{k}^{T} \\
& \times\left(\Sigma_{2}^{-1}-\Sigma_{k}^{-1}\right) x_{k} d x_{k}
\end{aligned}
$$

The last part in Eq.(29), $0.5 \int N\left(\mu_{k}, \Sigma_{k}\right) x_{k}^{T}\left(\Sigma_{2}^{-1}-\Sigma_{k}^{-1}\right) x_{k} d x_{k}$ can be evaluated as follows

$$
\begin{aligned}
& 0.5 \int N\left(\mu_{k}, \Sigma_{k}\right) x_{k}^{T}\left(\Sigma_{2}^{-1}-\Sigma_{k}^{-1}\right) x_{k} d x_{k} \\
& =0.5 \int N\left(\mu_{k}, \Sigma_{k}\right)\left[\left(x_{k}-\mu_{k}\right)^{T}\left(\Sigma_{2}^{-1}-\Sigma_{k}^{-1}\right)\left(x_{k}-\mu_{k}\right)\right. \\
& \left.+2 \mu_{k}^{T}\left(\Sigma_{2}^{-1}-\Sigma_{k}^{-1}\right) x_{k}-\mu_{k}^{T}\left(\Sigma_{2}^{-1}-\Sigma_{k}^{-1}\right) \mu_{k}\right] d x_{k} \\
& =0.5 \operatorname{tr}\left(\left(\Sigma_{2}^{-1}-\Sigma_{k}^{-1}\right) \Sigma_{k}\right)+0.5\left[A x_{k-1}+B u_{k-1}\right]^{T} \\
& \times\left(\Sigma_{2}^{-1}-\Sigma_{k}^{-1}\right)\left[A x_{k-1}+B u_{k-1}\right] \\
& =0.5\left(\operatorname{tr}\left[\Sigma_{2}^{-1} \Sigma_{k}\right]-n\right)+0.5\left[A x_{k-1}+B u_{k-1}\right]^{T} \\
& \times\left(\Sigma_{2}^{-1}-\Sigma_{k}^{-1}\right)\left[A x_{k-1}+B u_{k-1}\right]
\end{aligned}
$$

Substitute Eq.(30) back into Eq.(29), we have

$$
\beta_{1}\left(u_{k-1}, x_{k-1}\right)=0.5\left[A x_{k-1}+B u_{k-1}\right]^{T} \Sigma_{2}^{-1}\left[A x_{k-1}+B u_{k-1}\right]
$$

Similarly, $\beta_{2}\left(u_{k-1}, x_{k-1}\right)$ can be evaluated as follows

$$
\begin{aligned}
\beta_{2}\left(u_{k-1}, x_{k-1}\right) & =-\int s\left(x_{k} \mid u_{k-1}, x_{k-1}\right) \ln \left(\gamma\left(x_{k}\right)\right) d x_{k} \\
& =\int N\left(\mu_{k}, \Sigma_{k}\right)\left[0.5\left(x_{k}^{T} S_{k} x_{k}+\omega_{k}\right)\right] d x_{k} \\
& =0.5\left[A x_{k-1}+B u_{k-1}\right]^{T} S_{k}\left[A x_{k-1}+B u_{k-1}\right] \\
& +0.5 \bar{\omega}_{k}
\end{aligned}
$$

where

$$
\begin{aligned}
\bar{\omega} & =\operatorname{tr}\left(S_{k} \Sigma_{k}\right)+\omega_{k} \\
& =x_{k-1}^{T} M_{2} x_{k-1}+\omega_{k}
\end{aligned}
$$

with $M_{2}=D^{T} S_{k} \bar{Q} D$.

Therefore, using Eq.(31) and Eq.(32) in Eq.(23) and collecting the terms that multiply the control input, $u_{k-1}$ together yields,

$$
\begin{aligned}
\gamma\left(x_{k-1}\right) & =\int c^{I}\left(u_{k-1} \mid x_{k-1}\right) \exp \left[-\beta_{1}\left(u_{k-1}, x_{k-1}\right)\right. \\
& \left.-\beta_{2}\left(u_{k-1}, x_{k-1}\right)\right] d u_{k-1} \\
& =(2 \pi \Gamma)^{-\frac{1}{2}} \exp \left\{-0.5 x_{k-1}^{T}\left[A^{T}\left(\Sigma_{2}^{-1}+S_{k}\right) A\right.\right. \\
& \left.\left.+M_{2}\right] x_{k-1}-0.5 \omega_{k}\right\} \\
& \times \int \exp \left\{-0.5 u_{k-1}^{T}\left(B^{T}\left(\Sigma_{2}^{-1}+S_{k}\right) B+\Gamma^{-1}\right) u_{k-1}\right. \\
& \left.-x_{k-1}^{T} A^{T}\left(\Sigma_{2}^{-1}+S_{k}\right) B u_{k-1}\right\} d u_{k-1}
\end{aligned}
$$

Evaluating the integral in Eq.(34) we get

$$
\begin{aligned}
& \int \exp \left\{-0.5 u_{k-1}^{T}\left(B^{T}\left(\Sigma_{2}^{-1}+S_{k}\right) B+\Gamma^{-1}\right) u_{k-1}-x_{k-1}^{T}\right. \\
& \left.\times A^{T}\left(\Sigma_{2}^{-1}+S_{k}\right) B u_{k-1}\right\} d u_{k-1} \\
& =\exp \left\{0.5 x_{k-1}^{T} A^{T}\left(\Sigma_{2}^{-1}+S_{k}\right) B\left[B^{T}\left(\Sigma_{2}^{-1}+S_{k}\right) B+\Gamma^{-1}\right]^{-1}\right. \\
& \left.\times B^{T}\left(\Sigma_{2}^{-1}+S_{k}\right)^{T} A x_{k-1}\right\} \int \exp \left\{-0.5\left(u_{k-1}-x_{k-1}^{T} A^{T}\right.\right. \\
& \left.\times\left(\Sigma_{2}^{-1}+S_{k}\right) B\left[B^{T}\left(\Sigma_{2}^{-1}+S_{k}\right) B+\Gamma^{-1}\right]^{-1}\right)^{T} \\
& \times\left[B^{T}\left(\Sigma_{2}^{-1}+S_{k}\right) B+\Gamma^{-1}\right]\left(u_{k-1}-x_{k-1}^{T} A^{T}\left(\Sigma_{2}^{-1}+S_{k}\right)\right. \\
& \left.\left.\times B\left[B^{T}\left(\Sigma_{2}^{-1}+S_{k}\right) B+\Gamma^{-1}\right]^{-1}\right)\right\} d u_{k-1} \\
& =\exp \left\{0 . 5 x _ { k - 1 } ^ { T } A ^ { T } ( \Sigma _ { 2 } ^ { - 1 } + S _ { k } ) B \left[B^{T}\left(\Sigma_{2}^{-1}+S_{k}\right) B\right.\right. \\
& \left.\left.+\Gamma^{-1}\right]^{-1} B^{T}\left(\Sigma_{2}^{-1}+S_{k}\right)^{T} A x_{k-1}\right\} \\
& \times \ln \left(\pi^{\frac{1}{2}}\left(0.5\left[B^{T}\left(\Sigma_{2}^{-1}+S_{k}\right) B+\Gamma^{-1}\right]^{-\frac{1}{2}}\right)\right)
\end{aligned}
$$


Hence, we have

$$
\begin{aligned}
\gamma\left(x_{k}-1\right) & =\{2 \Gamma\}^{-\frac{1}{2}}\left(0.5\left[B^{T}\left(\Sigma_{2}^{-1}+S_{k}\right) B+\Gamma^{-1}\right]^{-\frac{1}{2}}\right) \\
& \times \exp \left\{0 . 5 x _ { k - 1 } ^ { T } \left(A ^ { T } ( \Sigma _ { 2 } ^ { - 1 } + S _ { k } ) B \left(B^{T}\right.\right.\right. \\
& \left.\times\left(\Sigma_{2}^{-1}+S_{k}\right) B+\Gamma^{-1}\right)^{-1} B^{T}\left(\Sigma_{k}^{-1}+b+S_{k}\right)^{T} A \\
& \left.\left.\left.-A^{T}\left(\Sigma_{k}^{-1}+b+S_{k}\right) A-M_{2}\right) x_{k-1}-0.5 \omega_{k}\right)\right\} \\
& =\exp \left\{0 . 5 x _ { k - 1 } ^ { T } \left[A^{T}\left(\Sigma_{2}^{-1}+S_{k}\right) B\right.\right. \\
& \times\left[B^{T}\left(\Sigma_{2}^{-1}+S_{k}\right) B+\Gamma^{-1}\right]^{-1} B^{T}\left(\Sigma_{2}^{-1}+S_{k}\right)^{T} \\
& \left.\times A-A^{T}\left(\Sigma_{2}^{-1}+S_{k}\right) A-M_{2}\right] x_{k-1} \\
& -0.5 \omega_{k}-0.5 \ln \left(0 . 5 \left(B^{T}\left(\Sigma_{2}^{-1}+S_{k}\right) B\right.\right. \\
& \left.\left.\left.+\Gamma^{-1}\right)\right)-0.5 \ln (2 \Gamma)\right\}
\end{aligned}
$$

Therefore the final result is given by

$$
\begin{aligned}
\gamma\left(x_{k}-1\right) & =\exp \left\{0 . 5 x _ { k - 1 } ^ { T } \left\{A ^ { T } ( \Sigma _ { 2 } ^ { - 1 } + S _ { k } ) B \left(B^{T}\left(\Sigma_{2}^{-1}+S_{k}\right) B\right.\right.\right. \\
& \left.+\Gamma^{-1}\right)^{-1} B^{T}\left(\Sigma_{2}^{-1}+S_{k}\right)^{T} A-A^{T}\left(\Sigma_{2}^{-1}+S_{k}\right) A \\
& \left.-2 M_{1}-M_{2}\right\} x_{k-1}+n-0.5 \omega_{k} \\
& \left.-0.5 \ln \left(0.5\left(B^{T}\left(\Sigma_{2}^{-1}+S_{k}\right) B+\Gamma^{-1}\right)\right)-0.5 \ln (2 \Gamma)\right\}
\end{aligned}
$$

Finding quadratic terms in $x_{k-1}$ gives the claimed form for $S_{k-1}$ that is specified in Eq.(20). The constant terms correspond to $\omega_{k-1}$ specified in Eq.(21). End of proof.

Following the above verification of the performance index, the next step is to evaluate the parameters of the optimal controller distribution such that the pdf of the states can follow the given pdf. Based on Eq.(15) and Eq.(19), we have the following theorem.

Theorem 2: The optimal controller minimising the performance index (14) is given by

$$
u_{k}=-K_{k} x_{k},
$$

where

$$
\begin{aligned}
K_{k} & =\left(\Gamma^{-1}+B^{T} M_{k} B\right)^{-1} B^{T} M_{k}^{T} A, \\
M_{k} & =\Sigma_{2}^{-1}+S_{k}
\end{aligned}
$$

Proof: Substituting Eq.(18), Eq.(31), Eq.(32) and Eq.(37) into Eq.(15) yields

$$
\begin{aligned}
c^{*}\left(u_{k-1} \mid x_{k-1}\right) & =\exp \left(-0.5\left\{u_{k-1}^{T}\left(\Gamma^{-1}+B^{T}\left(\Sigma_{2}^{-1}+S_{k}\right) B\right)\right.\right. \\
& \times u_{k-1}+2 x_{k-1}^{T} A^{T}\left(\Sigma_{2}^{-1}+S_{k}\right) B u_{k-1}+x_{k-1}^{T}\left(A^{T}\right. \\
& \times\left(\Sigma_{2}^{-1}+S_{k}\right) B\left[\Gamma^{-1}+B^{T}\left(\Sigma_{2}^{-1}+S_{k}\right) B\right]^{-1} B^{T} \\
& \left.\times\left(\Sigma_{2}^{-1}+S_{k}\right) A\right) x_{k-1}-\ln \left(0 . 5 \left[\Gamma^{-1}\right.\right. \\
& \left.\left.\left.\left.+B^{T}\left(\Sigma_{2}^{-1}+S_{k}\right) B\right)\right]-\ln (2 \Gamma)\right\}\right)
\end{aligned}
$$

Define $M_{k}=\Sigma_{2}^{-1}+S_{k}$, the equation can be expressed as

$$
\begin{aligned}
c^{*}\left(u_{k-1} \mid x_{k-1}\right) & =\exp \left(-0.5\left\{\left(u_{k-1}+\left(\Gamma^{-1}+B^{T} M_{k} B\right)^{-1}\right.\right.\right. \\
& \left.\times B^{T} M_{k}^{T} A x_{k-1}\right)^{T}\left(\Gamma^{-1}+B^{T} M_{k} B\right)\left(u_{k-1}\right. \\
& \left.+\left(\Gamma^{-1}+B^{T} M_{k} B\right)^{-1} B^{T} M_{k}^{T} A x_{k-1}\right) \\
& -x_{k-1}^{T} A^{T} M_{k} B\left(\Gamma^{-1}+B^{T} M_{k} B\right)^{-1} B^{T} M_{k}^{T} A \\
& \times x_{k-1}+x_{k-1}^{T}\left(A ^ { T } M _ { k } B \left[\left(\Gamma^{-1}+B^{T} M_{k} B\right)^{T}\right.\right. \\
& \left.\left.\times\left(\Gamma^{-1}+B^{T} M_{k} B\right)\right]^{-1} B^{T} M_{k} A\right) x_{k-1} \\
& \left.\left.-\ln \left(0.5\left(\Gamma^{-1}+B^{T} M_{k} B\right)\right)\right\}\right)
\end{aligned}
$$

It can be seen that the mean of the distribution given in Eq.(41) is the optimal control equation as Eq.(39). End of proof.

\section{Simulation}

To demonstrate the effectiveness of the derived novel algorithm which is based on the fully probabilistic design, the proposed algorithm is applied to the cart and inverted pendulum model used in [34]. The cart and pendulum model is shown to be described by the following discrete time dynamical equation. In order to highlight the positive features of our approach, we consider a different variant of the model in [34] where we add multiplicative noise part $D x_{k} v_{k}$ to their original model.

$$
x_{k+1}=A_{d} x_{k}+B_{d} u_{k}+D x_{k} v_{k}
$$

where

$$
\begin{gathered}
A_{d}=\left[\begin{array}{cccc}
1 & 0.1 & -0.0166 & -0.0005 \\
0 & 1 & -0.3374 & -0.0166 \\
0 & 0 & 1.0996 & 0.1033 \\
0 & 0 & 2.0247 & 1.0996
\end{array}\right], B_{d}=\left[\begin{array}{c}
0.0045 \\
0.0896 \\
-0.0068 \\
-0.1377
\end{array}\right] \\
D=\left[\begin{array}{cccc}
0.05 & 0.06 & 0.01 & 0.15 \\
0.01 & 0.115 & 0.16 & 0.07 \\
-0.1 & 0.2 & -0.005 & 0.115 \\
0.02 & -0.115 & -0.007 & 0.06
\end{array}\right]
\end{gathered}
$$

where $A_{d}, B_{d}$ represent the state and control matrices respectively which were assumed to have the same values as in [34]. The introduced matrix D that multiplies the noise term is created randomly. $v_{k}$ represents the Gaussian noise with zero mean and variance $Q=0.09$. The initial state $x_{0}$ of the inverted pendulum is assumed to start from $\left[\begin{array}{llll}0.02 & 0.1 & -0.1 & 0\end{array}\right]^{T}$. The ideal variance of controller $\Gamma$ is set to 0.5 since bigger variance of controller means faster converging speed. To achieve less randomness of states, the ideal covariance of state $\Sigma_{2}$ should be chosen small and the value we chose is shown as follows

$$
\Sigma_{2}=\left[\begin{array}{cccc}
0.04 & 0 & 0 & 0 \\
0 & 0.001 & 0 & 0 \\
0 & 0 & 0.0004 & 0 \\
0 & 0 & 0 & 0.03
\end{array}\right]
$$

To validate the performance of the randomised controller derived in this paper, the results are compared to the results obtained from the traditional FPD. As described in section II, the parameters of the system are estimated online using a neural network, and the control input was calculated based on the estimated parameters. The results of the simulation are shown in Fig 1 - Fig 6. Fig 1 - Fig 4 show the states of the system of the traditional FPD and our proposed method, from which it can be seen that all states converge to zero. Compared with the traditional FPD, the converging speed of the proposed method is clearly faster with less oscillation. The reason is that the proposed method is designed to deal with the multiplicative noise. Moreover, Fig 5 and Fig 6 display the control gain of the proposed algorithm and of the 
the traditional FPD, respectively. From Fig 5 and Fig 6, we can see that the gain values settle down after the parameter estimation converge. Therefore, the conclusion can be drawn that the proposed algorithm can achieve a better performance aiming at the system with multiplicative noise compared with the traditional FPD.

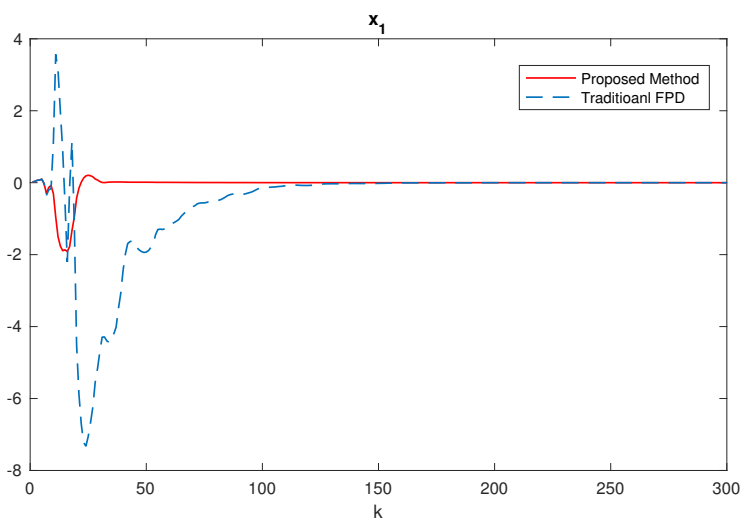

Fig. 1. State $x_{1}$

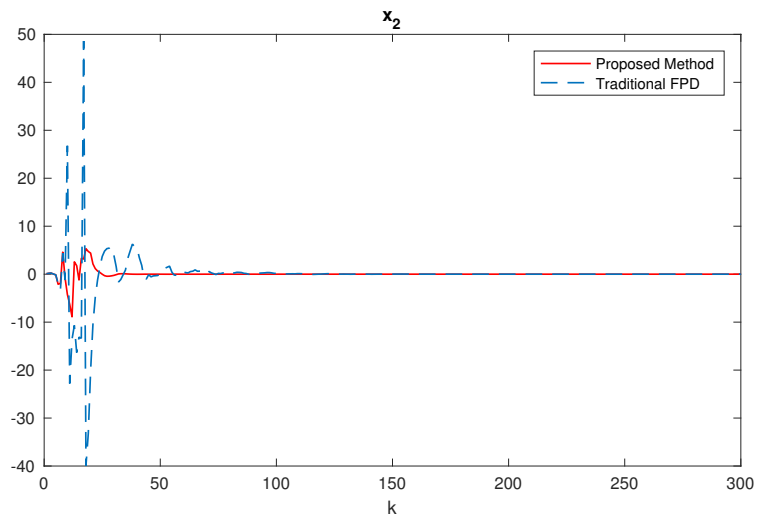

Fig. 2. State $x_{2}$

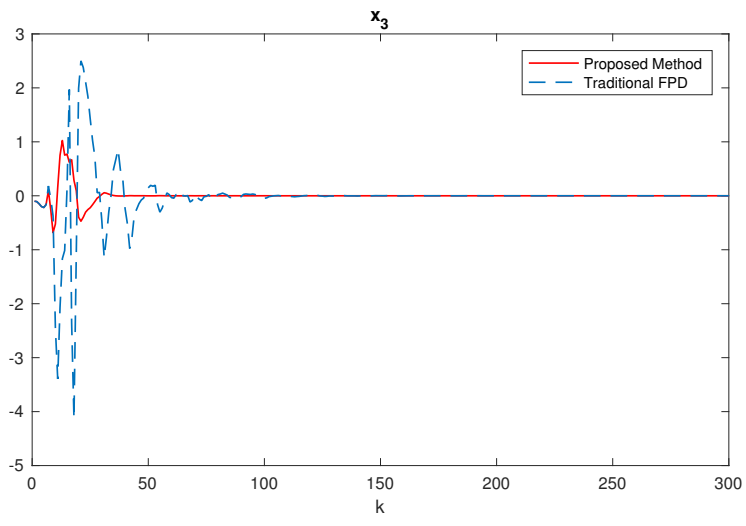

Fig. 3. State $x_{3}$

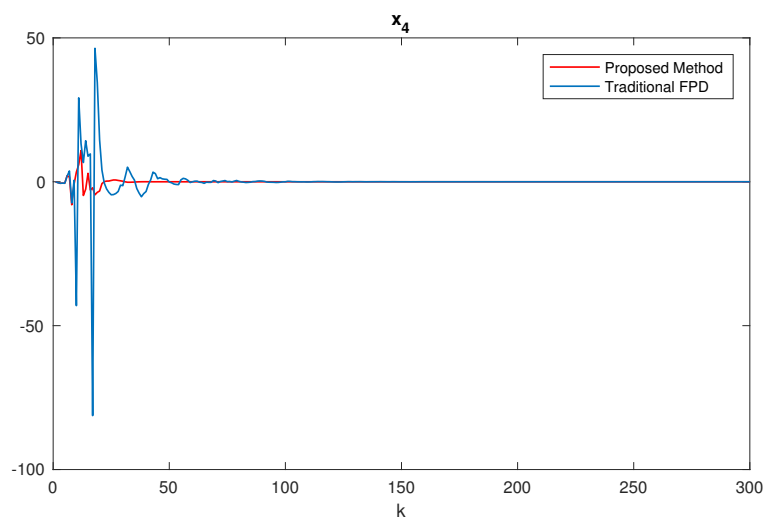

Fig. 4. State $x_{4}$

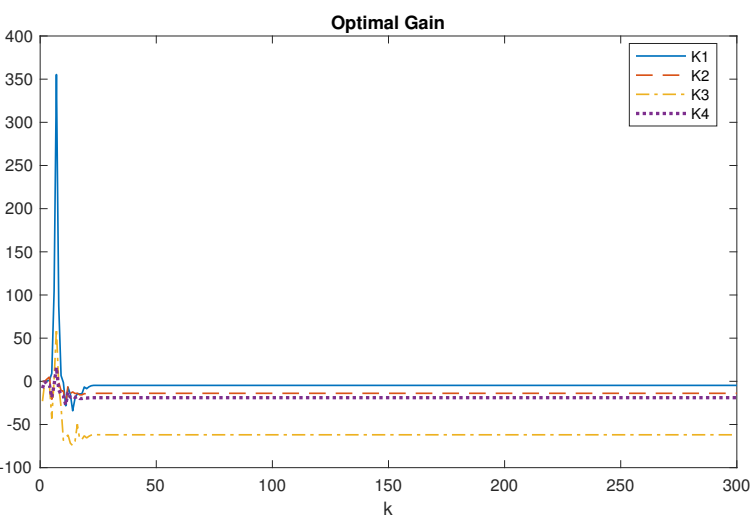

Fig. 5. Optimal gain $K$

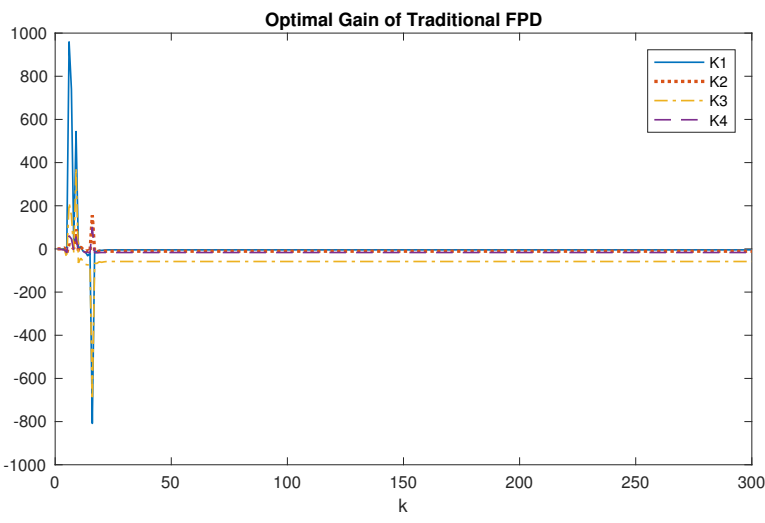

Fig. 6. Optimal gain $K$ of Traditional FPD

\section{CONCLUSIONS}

An FPD based method has been proposed in this paper for a class of linear stochastic dynamic systems with multiplicative noise. The linear optimisation has been applied to estimate the unknown parameters of the system and the performance index has been characterised by the KLD. Also, compared with the traditional FPD, the Raccatti equation has been modified to deal with the multiplicative noise part. Finally, the associated simulation results have been produced 
to verify the proposed control algorithm and compared with the results obtained from the traditional FPD.

\section{REFERENCES}

[1] R. M. Murray, K. J. Astrom, S. P. Boyd, R. W. Brockett, and G. Stein, "Future directions in control in an information-rich world," IEEE Control Systems, vol. 23, no. 2, pp. 20-33, 2003.

[2] M. Sain and S. Liberty, "Performance-measure densities for a class of lqg control systems," IEEE Transactions on Automatic Control, vol. 16, no. 5, pp. 431-439, 1971.

[3] Y. Zhou, Q. Zhang, and H. Wang, "Enhanced performance controller design for stochastic systems by adding extra state estimation onto the existing closed loop control," in Control (CONTROL), 2016 UKACC 11th International Conference on. IEEE, 2016, pp. 1-6.

[4] M. J. Grimble, "Controller performance benchmarking and tuning using generalised minimum variance control," Automatica, vol. 38, no. 12, pp. 2111-2119, 2002.

[5] J. Zhang, F. Zhang, and H. Wang, "Neural pid control strategy for superheated steam temperature based on minimum entropy," in Advanced Mechatronic Systems (ICAMechS), 2011 International Conference on. IEEE, 2011, pp. 524-528.

[6] Y. Zhou, Q. Zhang, H. Wang, P. Zhou, and T. Chai, "Ekf-based enhanced performance controller design for nonlinear stochastic systems," IEEE Transactions on Automatic Control, vol. 63, no. 4, pp. 1155-1162, 2018.

[7] H. Yue and H. Wang, "Minimum entropy control of closed-loop tracking errors for dynamic stochastic systems," IEEE Transactions on Automatic Control, vol. 48, no. 1, pp. 118-122, 2003.

[8] B. Chen and W. ZHANG, "Stochastic h2/h controlwith state-dependent noise," IEEE Transactions on Automatic Control, vol. 200, no. 4, p. 4.

[9] B.-S. Chen and W. Zhang, "Stochastic h/sub 2//h/sub/spl infin//control with state-dependent noise," IEEE Transactions on Automatic Control, vol. 49 , no. 1 , pp. $45-57,2004$.

[10] L. Guo and H. Wang, "Pseudo-pid tracking control for a class of output pdfs of general non-gaussian stochastic systems," in American Control Conference, 2003. Proceedings of the 2003, vol. 1. IEEE, 2003, pp. 362-367.

[11] H. Wang, "Control of conditional output probability density functions for general nonlinear and non-gaussian dynamic stochastic systems," IEE Proceedings-Control Theory and Applications, vol. 150, no. 1, pp. 55-60, 2003.

[12] L. Guo and L. Yin, "Robust pdf control with guaranteed stability for non-linear stochastic systems under modelling errors," IET control theory \& applications, vol. 3, no. 5, pp. 575-582, 2009.

[13] R. Herzallah, "Probabilistic dhp adaptive critic for nonlinear stochastic control systems," Neural Networks, vol. 42, pp. 74-82, 2013.

[14] R. Herzallah and M. Kárnỳ, "Fully probabilistic control design in an adaptive critic framework," Neural networks, vol. 24, no. 10, pp. 1128$1135,2011$.

[15] R. Herzallah, "Generalised probabilistic control design for uncertain stochastic control systems," Asian Journal of Control, 2018.

[16] M. Kárnỳ, "Towards fully probabilistic control design," Automatica, vol. 32, no. 12, pp. 1719-1722, 1996.

[17] A. Fel'dbaum, "Theory of dual control i," Avtomatika i Telemekhanika, vol. 21, no. 9, 1960.

[18] R. Herzallah, "Probabilistic dhp adaptive critic for nonlinear stochastic control systems," Neural Networks, vol. 42, pp. 74-82, 2013.

[19] R. Herzallah and M. Kárnỳ, "Towards probabilistic synchronisation of local controllers," International Journal of Systems Science, vol. 48, no. 3, pp. 604-615, 2017.

[20] C. M. Harris and D. M. Wolpert, "Signal-dependent noise determines motor planning," Nature, vol. 394, no. 6695, p. 780, 1998.

[21] E. Todorov and M. I. Jordan, "Optimal feedback control as a theory of motor coordination," Nature neuroscience, vol. 5, no. 11, p. 1226, 2002.

[22] E. Gershon, U. Shaked, and I. Yaesh, "H control and filtering of discrete-time stochastic systems with multiplicative noise," Automatica, vol. 37, no. 3, pp. 409-417, 2001.

[23] F. Wang and V. Balakrishnan, "Robust kalman filters for linear time-varying systems with stochastic parametric uncertainties," IEEE Transactions on Signal Processing, vol. 50, no. 4, pp. 803-813, 2002.

[24] W. Li, E. Todorov, and R. E. Skelton, "Estimation and control of systems with multiplicative noise via linear matrix inequalities," in American Control Conference, 2005. Proceedings of the 2005. IEEE, 2005, pp. 1811-1816.
[25] L. El Ghaoui, "State-feedback control of systems with multiplicative noise via linear matrix inequalities," Systems and Control Letters, vol. 24, no. 3, p. 223, 1995.

[26] S. E. Tuna, "Lqr-based coupling gain for synchronization of linear systems," arXiv preprint arXiv:0801.3390, 2008.

[27] F. Borrelli and T. Keviczky, "Distributed lqr design for identical dynamically decoupled systems," IEEE Transactions on Automatic Control, vol. 53, no. 8, pp. 1901-1912, 2008.

[28] Y. Cao and W. Ren, "Optimal linear-consensus algorithms: an lqr perspective," IEEE Transactions on Systems, Man, and Cybernetics, Part B (Cybernetics), vol. 40, no. 3, pp. 819-830, 2010.

[29] Q. Zhao and J. Jiang, "Reliable state feedback control system design against actuator failures," Automatica, vol. 34, no. 10, pp. 1267-1272, 1998.

[30] G. H. Golub and G. Meurant, Matrices, moments and quadrature with applications. Princeton University Press, 2009, vol. 30.

[31] V. Peterka, "Bayesian system identification," Automatica, vol. 17, no. 1 , pp. 41-53, 1981.

[32] D. P. Bertsekas, D. P. Bertsekas, D. P. Bertsekas, and D. P. Bertsekas, Dynamic programming and optimal control. Athena scientific Belmont, MA, 2005, vol. 1, no. 3 .

[33] B. Hall, Lie groups, Lie algebras, and representations: an elementary introduction. Springer, 2015, vol. 222.

[34] L. Zhang, Y. Shi, T. Chen, and B. Huang, "A new method for stabilization of networked control systems with random delays," IEEE Transactions on automatic control, vol. 50, no. 8, pp. 1177-1181, 2005. 